\title{
Real-time assessment of COVID-19 prevalence among multiple sclerosis patients: a multicenter European study
}

\author{
Gloria Dalla Costa ${ }^{1,2} \cdot$ Letizia Leocani ${ }^{1,2} \cdot$ Xavier Montalban $^{3} \cdot$ Ana Isabel Guerrero ${ }^{3} \cdot$ Per Soelberg Sørensen $^{4}$. \\ Melinda Magyari ${ }^{4} \cdot$ Richard J. B. Dobson ${ }^{5,6}$ - Nicholas Cummins ${ }^{7}$. Vaibhav A. Narayan ${ }^{8}$. Matthew Hotopf ${ }^{9}$. \\ Giancarlo Comi ${ }^{1}$ (D) $\cdot$ on behalf of the RADAR-CNS consortium
}

Received: 3 June 2020 / Accepted: 16 June 2020 / Published online: 2 July 2020

(C) Fondazione Società Italiana di Neurologia 2020

\begin{abstract}
We assessed the prevalence and impact of COVID-19 among multiple sclerosis (MS) patients across Europe by leveraging participant data collected as part of the ongoing EU IMI2 RADAR-CNS major programme aimed at finding new ways of monitoring neurological disorders using wearable devices and smartphone technology. In the present study, 399 patients of RADAR-MS have been included (mean age 43.9 years, $60.7 \%$ females) with $87 / 399$ patients (21.8\%) reporting major symptoms suggestive of COVID-19. A trend for an increased risk of COVID-19 symptoms under alemtuzumab and cladribine treatments in comparison to injectables was observed. Remote monitoring technologies may support health authorities in monitoring and containing the ongoing pandemic.
\end{abstract}

Keywords Multiple Sclerosis $\cdot$ Remote monitoring technologies $\cdot$ COVID-19

\section{Introduction}

SARS-CoV-2 is a coronavirus first identified in December 2019 in China as the cause of a cluster of pneumonia cases (COVID19 disease) of previously unknown aetiology [1]. The virus has since spread globally, with the World Health Organization (WHO) declaring a pandemic state in March 2020 (https:// www.who.int/emergencies/diseases/novel-coronavirus-2019/

Electronic supplementary material The online version of this article (https://doi.org/10.1007/s10072-020-04519-x) contains supplementary material, which is available to authorized users.

Giancarlo Comi

comi.giancarlo@hsr.it

1 Institute of Experimental Neurology, IRCCS Ospedale San Raffaele, via Olgettina 60, 20132 Milan, Italy

2 Neurorehabilitation Unit, IRCCS Ospedale San Raffaele, Milan, Italy

3 Multiple Sclerosis Centre of Catalonia (Cemcat), Department of Neurology/Neuroimmunology, Hospital Universitari Vall d'Hebron, Universitat Autònoma de Barcelona, Barcelona, Spain

4 Danish Multiple Sclerosis Centre, Department of Neurology, Copenhagen University Hospital Rigshospitalet, Copenhagen, Denmark situation-reports). However, the real prevalence and impact of the disease are unknown.

Remote Assessment of Disease and Relapse in Central Nervous System Disorders (RADAR-CNS) is an Innovative Medicine Initiative-funded project aimed at monitoring major depressive disorders, epilepsy, and multiple sclerosis (MS) using wearable devices and smartphone technology [2]. Through the ongoing remote monitoring of MS patients in

5 The Department of Biostatistics and Health informatics, Institute of Psychiatry, Psychology and Neuroscience, King's College London, London, UK

6 Institute of Health Informatics, University College London, London, UK

7 Embedded Intelligence for Health Care \& Wellbeing, University of Augsburg, Augsburg, Germany

8 Janssen Research and Development, LLC, Titusville, NJ, USA

9 The Department of Psychological Medicine, Institute of Psychiatry, Psychology and Neuroscience, King's College London, London, UK 
Italy, Spain, and Denmark, we aimed to explore the prevalence and impact of the current pandemic among MS patients across Europe.

\section{Methods}

Study participants The MS work package of RADAR-CNS started recruiting in June 2018 with the aims to assess, through remote monitoring, the impact of MS on the emotional state of recently (< year) diagnosed patients and to monitoring disability and fatigue on relapsing-remitting (RRMS) and secondary progressive (SPMS) patients. Since then, passive (a smartwatch device and mobile sensors) and active monitoring strategies (questionnaires, cognitive, and physical tests) have been in place. Figure S1 shows the full inclusion criteria and assessment schemes. Approval of the protocol was obtained from hospitals' ethical committees and informed consent obtained from all patients.

COVID-19 data A questionnaire to collect data systematically on COVID-19-related adverse events (Fig. S2) was sent to patients twice at a two-weekly interval (on March 25, 2020, and April 8, 2020, and data collected until April 15, 2020), through the RADAR-CNS active monitoring system. The results are based on the combination of the surveys. All data was managed by the open-source RADAR-based platform (www.radar-base.org) [2].

COVID-19 case definitions WHO guidelines define suspected cases as patients with fever, signs/symptoms of respiratory disease and travels/residence in a location reporting community transmission of COVID-19, or in contact with confirmed/ probable COVID-19 cases in the 14 days prior to symptom onset, or people requiring hospitalization in the absence of alternative diagnosis [3]. As most of the confirmed COVID-19 cases do not fulfill these criteria [4] and considering the questionnaire was performed in areas with known community transmission of COVID-19, two alternative definitions were used: patients experiencing fever or anosmia/ageusia + any other COVID-19 symptoms, or respiratory symptoms + two other COVID-19 symptoms (case definition 1), and patients experiencing fever + any other COVID-19 symptoms, or respiratory symptoms + anosmia/ageusia (case definition 2). Laboratory-confirmed cases were included in both case definitions.

Statistical analysis Univariate and multivariate penalized likelihood logistic regression models (Firth method) have been used to assess predictors of COVID-19 occurrence [5]. The impact of MS treatments on COVID-19 risk was first evaluated separately for each drug (Fig. S3) and then analyzed according to the following treatment groups: injectables (interferons and glatiramer acetate), oral treatments (dimethyl fumarate, teriflunomide), fingolimod, natalizumab, antiCD20 (ocrelizumab), immune system resetting drugs (alemtuzumab, cladribine). $p$ values $<0.05$ were deemed statistically significant. Computing environment $\mathrm{R}$ was used for all statistical analyses (R Development Core Team, 2005).

\section{Results}

\section{Cohort baseline characteristics (Table 1)}

As of March 1, 2020, 499 patients have been enrolled in the RADAR-MS studies and 403 (81\%) remained under active and passive remote monitoring. The COVID-19 questionnaire was completed at least once by 399 patients $(99 \%)$.

Table 1 Baseline characteristics of the study cohort

\begin{tabular}{ll}
\hline Characteristic & $n=399$ \\
\hline Age, years & $43.9 \pm 10.7$ \\
Sex, $n(\%)$ & \\
Females & $242(60.7)$ \\
Males & $157(39.3)$ \\
Smokers & $84(21.1)$ \\
BMI & $24.47 \pm 5.7$ \\
Healthcare workers & $19(4.7)$ \\
Course of disease, $n(\%)$ & \\
Clinically isolated syndromes & $5(1.3)$ \\
Relapsing-remitting & $305(76.4)$ \\
Secondary progressive & $89(22.3)$ \\
Disease duration, years & $13.1 \pm 10.3$ \\
Expanded Disability Status Scale & $3.5(2.0-4.0)$ \\
Presence of comorbidities, $n(\%)$ & $144(36.1)$ \\
Cardiovascular/hypertension & $51(12.8)$ \\
Diabetes & $5(1.3)$ \\
COPD & $1(0.3)$ \\
Malignancies & $2(0.5)$ \\
Ongoing MS treatments, $n(\%)$ & $359(90)$ \\
Interferons/glatiramer acetate & $81(20.3)$ \\
Dim. fumarate/teriflunomide & $108(27.1)$ \\
Natalizumab & $44(11.0)$ \\
Fingolimod & $67(16.8)$ \\
Anti-CD20 & $44(11.0)$ \\
Alemtuzumab/cladribine & $15(3.8)$ \\
Vitamin D supplements & $237(59.4)$ \\
ACE inhibitors & $40(10.0)$ \\
\hline
\end{tabular}

$B M I$, body mass index; $C O P D$, chronic obstructive pulmonary disease; $M S$, multiple sclerosis; $D M D$, disease-modifying drugs; mean \pm standard deviation; number (percentage) 


\section{Prevalence and predictors of COVID-19 by case definition}

Major symptoms suggestive of COVID-19 infection have been reported by 87 patients $(21.8 \%, 95 \%$ CI $17.8-25.9)$. Among these, $31(7.8 \%)$ had a fever, $73(18.3 \%)$ had respiratory symptoms, $38(9.5 \%)$ had gastrointestinal disturbances, 17 (4.3\%) had conjunctivitis, and 17 (4.3\%) experienced ageusia and anosmia. Two patients had COVID-19 pneumonia, and one patient was hospitalized. No one died of COVID-19.

In the cohort, 52 patients $(13.0 \%, 95 \%$ CI 9.7-16.3) met the case definition 1 , and $37(9.3 \%, 95 \%$ CI 6.4-12.1) met the case definition 2 criteria.

Ten patients were tested for SARS-CoV-2 through nasopharyngeal swab: four of them were positive, and one tested negative but had COVID-19 pneumonia upon X-ray examination and swab test which was performed 26 days after symptom onset. Figure $\mathrm{S} 4$ shows the occurrence of suspected COVID-19 cases.

Patients with SPMS were at lower risk than relapsingremitting MS of fulfilling either definitions (case definition 1: OR $0.36(0.13-0.84)$; case definition 2: OR 0.23 (0.05$0.71), p<0.01$ for all cases, Table 1). A trend for patients on cladribine/alemtuzumab to be more likely classified as COVID-19 cases than patients with injectables was observed, independently of age, sex, and disease course (case definition 1: OR 3.78 (1.00-15.93), $p$ 0.05; case definition 2: OR 4.87 (1.10-21.04), $p$ 0.04, Fig. 1, Table S2).

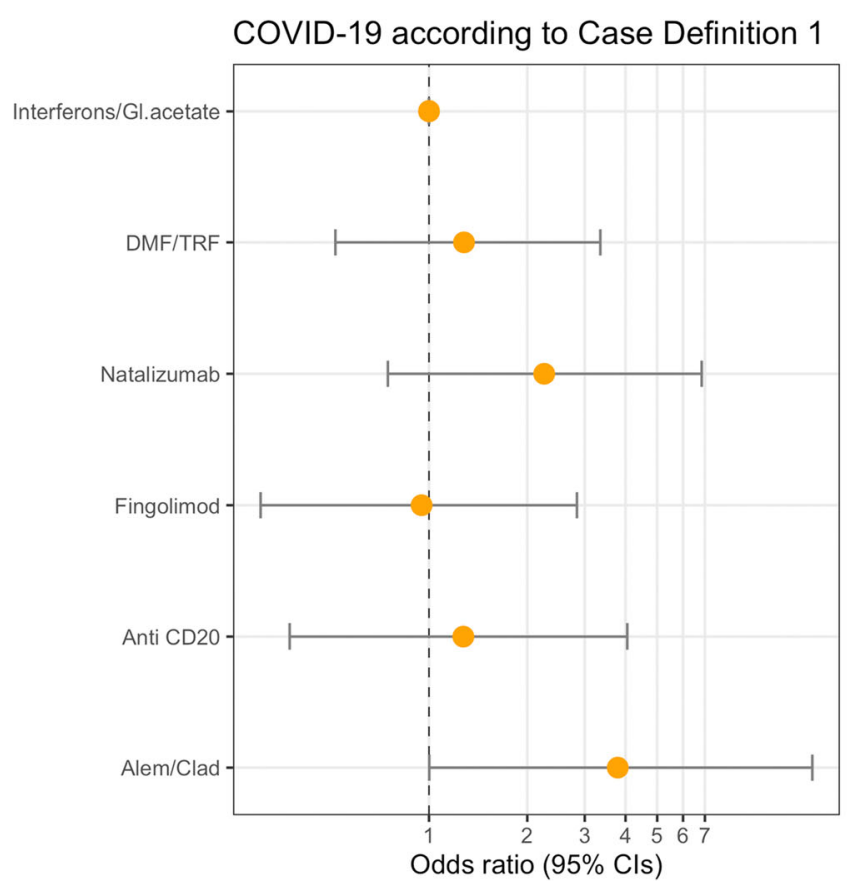

Odds ratios (yellow points) and 95\% confidence intervals (horizontal bars) have been estimated through the use of logistic regression models with a penalized likelihood method for rare events (Firth method), adjusted for age, sex, and course of the disease. Case definition 1: patients experiencing fever/anosmia/ageusia and any other COVID-19 symptoms or cough/dyspnea and two other COVID-19 symptoms; case definition 2: patients experiencing fever and any other COVID-19 symptoms or cough/dyspnea and anosmia/ ageusia. Patients with a laboratory-confirmed diagnosis of COVID-19 were also considered cases for both definitions.

\section{Impact of COVID on MS patients}

Changes in daily activities because of COVID-19 were reported by 344 patients (86\%): 148 (37\%) started working from home, $66(17 \%)$ took care of children, and four (1\%) cared for a sick relative. No outdoor hobbies or social activities were reported by 228 patients (57\%), and the daily average time spent outside was $1.8 \mathrm{~h}$. On the emotional level, 132 patients (33\%) were deeply worried by the pandemic, 233 (58\%) moderately worried, and $32(8 \%)$ were unaffected.

\section{Discussion}

A number of newly diagnosed COVID-19 cases are updated daily (https://www.who.int/emergencies/diseases/novel-coronavirus-2019/situation-reports), but these are clearly

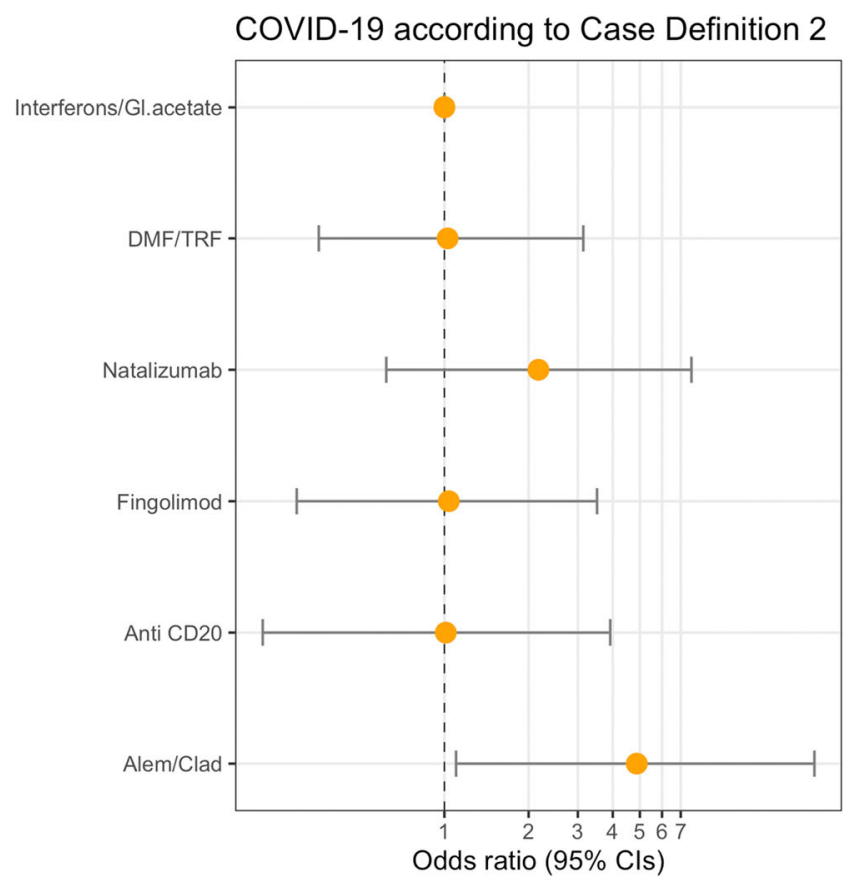

Fig. 1 Disease-modifying drugs and risk of suspected COVID-19 infection diagnosis. Gl. acetate glatiramer acetate; DMF, dimethyl fumarate; TRF, teriflunomide; Alem, alemtuzumab; Cladr, cladribine 
underestimated [6] as diagnosis depends on local availability of the test. In our study, five confirmed COVID-19 cases (four with positive swab tests and one patient with COVID-19 pneumonia) were reported (1.3\%); a low prevalence as just ten patients $(2.5 \%)$ of the cohort were tested for the virus. Using different case definitions, the percentage of MS patients with symptoms suggestive of infection varied between 9 and $13 \%$. These figures align with a recent study suggesting that the prevalence of the disease in Europe, in particular Italy, maybe nine times higher than officially reported [6]. According to our results, there is no evidence of a worse COVID-19 evolution in patients affected by MS: two patients only developed pneumonia and one was hospitalized. Global data indicates that morbidity is lower in younger COVID-19 patients [7], as our data also suggests. Patients with SPMS had a lower risk of infection compared with RRMS, maybe because they are less treated and with less-frequent social contacts. Disease-modifying treatments did not significantly change the risk and severity of COVID-19; however, a trend for an increased risk of infection with immune-resetting drugs (alemtuzumab and cladribine) was observed compared with injectables, despite low numbers prevent any definite conclusion. This aligns with previous studies showing a generally increased risk of infections with cladribine and alemtuzumab $[8,9]$. The COVID-19 pandemic has had a heterogeneous impact in different countries. Our patients come from highly affected areas in Europe; therefore, our results may be partially representative of the general MS population. However, the study highlights the importance of remote monitoring technologies that have shed some light on the impact of COVID-19 in our MS patients in real time and may provide support to health authorities in monitoring and containing the pandemic.

Acknowledgments We thank Maria Libera La Porta, Matteo Martinis, and Lucia Roselli for their support to the study

Author's contributions G.D.C., A.I.G., P.S.S., M.M., N.C., V.A.N., M.H., and G.C. contributed to the study design. G.D.C. contributed to the data analysis, figures drawing, and manuscript writing. L.L., X.M., A.I.G., P.S.S., M.M., N.C., R.J.B.D., V.A.N., M.H., and G.C. contributed to the critical revision of the manuscript. R.J.B.D. contributed to the platform design and implementation. X.M., P.S.S., N.C., R.J.B.D., V.A.N., M.H., and G.C. contributed to the administrative, technical, and clinical support of the study. G.D.C., A.I.G., and M.M. contributed to data collection.

Funding information This study was supported by EU/EFPIA IMI Joint Undertaking 2 (RADAR-CNS grant no. 115902).

Compliance with ethical standards Approval of the protocol was obtained from hospitals' ethical committees and informed consent obtained from all patients.
Conflict of interest VAN is an employee of Janssen Research \& Development LLC and may own equity in the company.

Disclaimer This communication reflects the views of the RADAR-CNS consortium, and neither IMI nor the European Union and EFPIA are liable for any use that may be made of the information contained herein.

Data sharing The scientific data used in this paper is collected under the project: EU/EFPIA IMI RADAR-CNS (grant agreement no. 115902). The data usability and sharing are performed under the agreed guidelines of the RADAR-CNS consortium. The anonymized data will be shared upon request subject to RADAR-CNS consortium approval. Pending the aforementioned approval, data sharing will be made in a secure setting, on a per-case-specific manner. Please submit such requests to the corresponding author of the paper. The shared data cannot be used for publication purposes without the explicit consent of the RADAR-CNS consortium. The additional related documents are available (e.g., study protocol, statistical analysis plan, informed consent form) upon request.

\section{References}

1. Zhou P, Yang XL, Wang XG et al (2020) A pneumonia outbreak associated with a new coronavirus of probable bat origin. Nature 579(7798):270-273

2. Ranjan Y, Rashid Z, Stewart C et al (2019) RADAR-base: open source mobile health platform for collecting, monitoring, and analyzing data using sensors, wearables, and mobile devices. JMIR Mhealth Uhealth 7(8):e11734

3. World Health Organization (2020) Surveillance case definitions for human infection with novel coronavirus $(\mathrm{nCoV})$ : interim guidance v1, January 2020. World Health Organization. https://apps.who.int/ iris/handle/10665/330376. License: CC BY-NC-SA 3.0 IGO. Accessed 1 Jul 2020

4. Huang C, Wang Y, Li X et al (2020) Clinical features of patients infected with 2019 novel coronavirus in Wuhan, China. Lancet 395(10223):497-506

5. Firth D (1993) Bias reduction of maximum likelihood estimates. Biometrika [Internet]. 80(1):27-38

6. Lachmann A, Jagodnik KM, Giorgi FM, Ray F Correcting underreported COVID-19 case numbers: estimating the true scale of the pandemic. medRxiv 2020.03.14.20036178. https://doi.org/10.1101/ 2020.03.14.20036178

7. Republic of Korea Center of Disease Control. Updates on COVID19 in Republic of Korea, 3/13/2020.

8. Giovannoni G, Comi G, Cook S et al (2010) A placebo-controlled trial of oral cladribine for relapsing multiple sclerosis. N Engl J Med 362:416-426

9. Cohen JA, Coles AJ, Arnold DL et al (2012) Alemtuzumab versus interferon beta 1a as first-line treatment for patients with relapsingremitting multiple sclerosis: a randomised controlled phase 3 trial. Lancet 380:1819-1828

Publisher's note Springer Nature remains neutral with regard to jurisdictional claims in published maps and institutional affiliations. 